\title{
Problematic use of prescription-type opioids prior to heroin use among young heroin injectors
}

\author{
This article was published in the following Dove Press journal: \\ Substance Abuse and Rehabilitation \\ II October 201। \\ Number of times this article has been viewed
}

\author{
Robin A Pollini' \\ Caleb J Banta-Green ${ }^{2}$ \\ Jazmine Cuevas-Mota ${ }^{3}$ \\ Mitcheal Metzner ${ }^{3}$ \\ Eyasu Teshale ${ }^{4}$ \\ Richard S Garfein ${ }^{3}$ \\ 'Pacific Institute for Research \\ and Evaluation, Calverton, MD; \\ ${ }^{2}$ Alcohol and Drug Abuse Institute, \\ University of Washington, Seattle, WA; \\ ${ }^{3}$ Department of Medicine, University \\ of California San Diego, La Jolla, CA; \\ ${ }^{4}$ National Center for HIVIAIDS, Viral \\ Hepatitis, STD and TB Prevention, \\ Centers for Disease Control and \\ Prevention, Atlanta, GA, USA
}

Background: Misuse of prescription-type opioids and related adverse health effects are increasing, but little is known about the role of these drugs as a precursor to heroin use. We conducted an exploratory study to determine the proportion of young heroin injectors reporting problematic use of prescription-type opioids prior to using heroin, and to describe the factors associated with prior problematic prescription-type opioid use.

Methods: Between March 2009 and June 2010, we recruited injection drug users (IDUs) for a crosssectional study of hepatitis $C$ virus infection risk. Participants were aged $18-40$ years and had injected illicit drugs within the previous six months. A computerized self-administered survey assessed sociodemographics, drug use history, human immunodeficiency virus (HIV)/hepatitis C virus risk behaviors and perceptions, and medical history. We added questions on prescription-type opioid use to the parent study in March 2010; heroin injectors who subsequently enrolled and reported problematic prescription-type opioid use prior to heroin initiation were compared with other heroin IDUs using univariate and multivariate regression methods.

Results: Among 123 heroin IDUs, 49 (39.8\%) reported problematic prescription-type opioid use prior to heroin initiation ("prescription-type opioid first injection drug users" [PTO-First IDUs]). PTO-First IDUs had higher odds of injecting with friends (adjusted odds ratio [AOR] 6.01; 95\% confidence interval [CI] 1.90-19.07), getting new syringes from a spouse/family member/sex partner (AOR 23.0; 95\% CI 2.33-226.0), knowing about the local syringe exchange program (AOR 7.28; 95\% CI 1.17-45.05), using powder cocaine (AOR 3.75; 95\% CI 1.43-9.86), and perceiving themselves as less likely than other IDUs to get HIV (AOR 4.32; 95\% CI 1.26-14.77). They had lower odds of ever being tested for HIV (AOR 0.25; 95\% CI 0.08-0.80).

Conclusion: A high proportion of young heroin IDUs reported problematic prescription-type opioid use prior to initiating heroin use. Our study provides several avenues for future investigation to help further characterize this subset of IDUs and their risks and perceptions related to HIV and other blood-borne pathogens.

Keywords: injection drug users, prescription-type opioids

\section{Background}

Prescription-type opioids, eg, oxycodone, hydrocodone, and morphine, play an important role in the medical management of pain, but the nonmedical use and abuse of these drugs is a topic of growing concern in the United States. In 2009, 5.3 million $(2.1 \%)$ persons aged $\geq 12$ years reported nonmedical use of pain relievers in the previous month, and one-third (35\%) met the criteria for dependence or abuse according to the US National Survey on Drug Use and Health; the highest prevalence was among those aged 18-25 years (4.8\%) followed by those aged $12-17$ years $(2.7 \%){ }^{1}$ The school-based Monitoring the Future survey found that $3.6 \%$ of US twelfth graders
Correspondence: Robin A Pollini Pacific Institute for Research and Evaluation, I I 720 Beltsville Drive, Suite 900 , Calverton, MD 20705, USA

$\mathrm{Tel}+$ I 30 I 7552755

Email rpollini@pire.org 
engaged in nonmedical use of prescription pain relievers in the previous month, compared with $3.0 \%$ in 2001, which was a statistically significant increase. ${ }^{2}$

Public health surveillance indicates that adverse health effects associated with prescription-type opioid misuse have also increased substantially over the last decade. The proportion of persons entering drug treatment who cited a prescription opioid as their primary drug of abuse increased from $1.5 \%$ in 2000 to $6.8 \%$ in $2009,{ }^{3}$ and drug-related emergency department visits involving pain relievers rose from 49.4 per 100,000 population in 2004 to 111.6 per 100,000 in 2009. ${ }^{4}$ Further, from 1999 to 2007, the number of US poisoning deaths involving any opioid analgesic more than tripled from 4041 to 14,459 , accounting for $36 \%$ of total poisoning deaths in 2007 compared with $20 \%$ in 1999.5

Despite substantial concern regarding the misuse of prescription-type opioids and their adverse effects on health, much less attention has been paid to the potential of prescription-type opioids to serve as a precursor to heroin use. The first mention of transitions from prescription-type opioids to heroin in the scientific literature was a letter to the editor of American Family Physician in 2003 by Siegal et al. ${ }^{6}$ The letter described an Ohio-based case series of 10 recently initiated young heroin users, of whom five reported abusing prescription-type opioids (particularly OxyContin ${ }^{\mathrm{TM}}$ ) prior to their first heroin use. Subjects reported eventually developing tolerance to prescription-type opioids and experiencing withdrawal symptoms when the drugs were unavailable. They noted that heroin was both more readily available and less expensive than OxyContin and that "they would have never tried heroin had they not become addicted to OxyContin". The authors also reported that they "continually identify persons who report a similar progression from prescription opioid use to heroin injection". A later qualitative study conducted by a related research group in Ohio documented similar transitions from heavy prescription-type opioid use to heroin among "new-generation users" aged 19-23 years.

Inciardi et al similarly documented transitions from prescription-type opioid use to heroin in a 2004 ultrarapid assessment of prescription opioid abuse and diversion in Wilmington, Delaware. In six focus groups involving a total of 32 treatment-based prescription-type opioid users, more than four-fifths reported a history of heroin use and the "the vast majority" said that prescription-type opioid abuse preceded their first use of heroin. Similar to the reports from Ohio, these focus group participants reported that their transition from prescription-type opioids to heroin was attributed to the affordability of heroin relative to the high street cost of prescription-type opioids. The authors concluded that "most [participants] reported that prescription opioids were indeed their gateway to heroin use".

Most recently, Lankenau et $\mathrm{al}^{9}$ described findings from a mixed methods study of 50 young IDUs in Los Angeles and New York who reported misuse of any prescription drug in the previous three months. All of the study participants had misused prescription-type opioids and used heroin, and $86 \%$ misused prescription-type opioids prior to initiating heroin use. The authors note that these findings corroborate those by Siegal et $\mathrm{al}^{6}$ and Inciardi et $\mathrm{al}^{8}$ that prescription-type opioid misuse may constitute an important first step on the pathway toward heroin use.

The aforementioned articles constitute the bulk of the scientific literature on prescription-type opioid misuse prior to heroin use. Of particular concern is the transition to heroin injection, which puts users at risk of infection with blood-borne pathogens like human immunodeficiency virus (HIV) and hepatitis $\mathrm{C}$ virus (HCV) in addition to increasing their risk of drug overdose due to the variable and unknown potency of heroin. We undertook a small, unfunded pilot study to explore problematic use of prescription-type opioids prior to heroin use among current IDUs with a history of heroin injection. The aims of this exploratory study were to determine the proportion of young heroin injectors who experienced problematic prescription-type opioid use prior to using heroin and to describe the factors associated with prior problematic prescription-type opioid use. Our goal was to characterize this as yet unelucidated subpopulation of IDUs to understand whether and how they differ from other heroin IDUs and explore the implications of these findings for HIV and HCV prevention efforts.

\section{Methods}

Our analysis was nested in a cross-sectional study of HIV and HCV risk behaviors among young IDUs in San Diego, California. ${ }^{10}$ The purpose of the parent study was to evaluate three different methods of injection drug user recruitment to determine the optimal method for ongoing national $\mathrm{HCV}$ surveillance. The study was funded by the Centers for Disease Control and Prevention and the protocol was approved by an institutional review board at the University of California, San Diego.

Enrollment for the parent study began in March 2009 and continued through June 2010. Eligibility criteria were: age 18-40 years; injecting illicit drugs within the previous six months (confirmed by physical inspection of track marks); residing in San Diego; agreeing to a blood draw for $\mathrm{HCV}$ 
and HIV testing; agreeing to provide contact information; and being willing to give informed consent. In March 2010 , approximately one year after the study began, we added questions to the survey instrument to explore prior problematic prescription-type opioid use among heroin injectors. Of 566 IDUs enrolled in the parent study, 415 had ever injected heroin; 123 of these 415 heroin injectors (29.6\%) enrolled after the questions regarding prescription-type opioid use were added and were thus eligible for our exploratory analysis. These 123 heroin IDUs did not differ significantly from the 292 heroin IDUs enrolled earlier in the study with regard to gender, age, race, ethnicity, or educational attainment.

\section{Recruitment}

The three methods of recruitment were street outreach, venue-based recruitment, and respondent-driven sampling. Street outreach involved street intercepts with IDUs in known high drug use neighborhoods. Outreach workers distributed recruitment cards and posted flyers in bars, clubs, coffee houses, and other establishments frequented by IDUs. Current study participants were also encouraged to inform their peers about the study. Venue-based recruiting consisted of inviting syringe exchange program clients to participate in the study when they came to exchange syringes. Syringe exchange program clients were referred to a study van parked adjacent to the syringe exchange program or to the study office. Respondent-driven sampling is a chain referral sampling approach that uses mathematical modeling to produce unbiased prevalence estimates. ${ }^{11} \mathrm{~A}$ diverse group of initial participants or "seeds" (heterogeneous by age, gender, and neighborhood) was selected and given uniquely coded coupons to refer their peers to the study. Waves of recruitment continued as subjects returning with their coupons were each given coupons to recruit other members of their own social networks.

\section{Data collection}

Study participants completed a quantitative questionnaire using audio computer-assisted self interview technology. The questionnaire covered sociodemographics, injection and non-injection drug use history (past three months), HIV/HCV risk behaviors (past three months), perceptions of $\mathrm{HIV} / \mathrm{HCV}$ risk, medical history, and health care utilization. Questions regarding HIV/HCV risk perceptions were worded as follows: "Compared to other drug users in the San Diego area, how likely do you think you are to get infected with [HIV/AIDS, Hepatitis C]?" with five possible responses, ie, much more likely, a bit more likely, about the same, a bit less likely, and much less likely. For the purposes of analysis, these responses were condensed into three categories, ie, more likely, about the same, and less likely.

Participants enrolled after the prescription-type opioid questions were added to the parent study, and those who reported ever injecting heroin, either alone or in combination with other drugs, were asked "Before you began using heroin, were you hooked on prescription-type opioids?" Those who answered affirmatively were then asked "Which prescription-type opioids were you hooked on?" and given a list of prescription-type opioids as well as an "other" option. The wording of these questions was identical to that used in surveys conducted at syringe exchange programs in Seattle, Washington, and subsequently in Portland, Oregon, to allow for comparison across the three regions. ${ }^{12,13}$ All interviews were completed by the participant in a private area (ie, office, clinical examination room, mobile van) where their responses were not visible to study staff.

\section{Statistical analyses}

Descriptive statistics were used to quantify and characterize problematic prescription-type opioid use prior to initiating heroin use. Characteristics of heroin injectors who responded affirmatively to the question about prescription-type opioid use ("PTO-First IDUs") were compared with the characteristics of those who responded negatively using Chi-square tests for categorical variables and $t$-tests and the Wilcoxon rank sum test for normally and non-normally distributed continuous variables, respectively. Variables that achieved a significance level of $P<0.20$ were tested in a multiple logistic regression model. We chose this more conservative significance level so that variables that were independently associated with PTO-First IDUs status but did not achieve $P<0.05$ in the univariate analysis due to our small sample size would be tested in the regression model. Each variable was added into the regression model in a manual forward stepwise fashion using PTO-First IDUs status (yes/no) as the binary outcome variable. Specifically, we entered one variable into the regression model at a time beginning with the variable with the lowest $P$ value and proceeding in order to the variable with the highest $P$ value (up to $P<0.20$ ). The likelihood ratio test was used to compare nested models to determine which variables were retained in the final model at a significance level $<5 \%$. We tested for interactions across variables in the final model, as well as for interactions by gender. We also controlled for recruitment method to address any differences in the association between prior prescription-type opioid use and exposures of interest by recruitment type. 


\section{Results}

Of the 123 heroin IDUs who responded to questions regarding prior problematic prescription-type opioid use, $14.6 \%$ were recruited using respondent-driven sampling, 48.0\% through venue-based recruiting, and $37.4 \%$ through street outreach. There was no significant difference in recruitment method based on PTO-First IDUs status. Seventy-five percent of the sample was male, 53\% white non-Hispanic, 28\% Hispanic, and $19 \%$ other/mixed race. Median age was 29 years (interquartile range 22-32) and median time since first injection was seven years (interquartile range 2-11). Overall, 49 (39.8\%) reported problematic prescription-type opioid use prior to their first heroin use (ie, PTO-First IDUs). The median number of prescription-type opioids these IDUs reported using was three (interquartile range 1-4), including Oxycontin/oxycodone (75.5\%), Vicodin ${ }^{\mathrm{TM}} /$ hydrocodone (69.4\%), morphine (34.7\%), Percocet ${ }^{\mathrm{TM}}(20.4 \%)$, fentanyl $(20.4 \%)$, Dilaudid ${ }^{\mathrm{TM}} /$ hydromorphone $(16.3 \%)$, methadone $(14.3 \%)$, Demerol ${ }^{\mathrm{TM}}$ (8.2\%), and other prescription-type opioids (4.1\%).

Table 1 compares PTO-First IDUs and other heroin IDUs with regard to demographics and history of drug use
(Table 1). A higher proportion of PTO-First IDUs were white non-Hispanic, completed some schooling beyond high school, and lived with their parents during the past six months. Their drug injection histories also differed from other heroin IDUs in that they were significantly older at the time of first injection, but initiated injection much more recently.

Table 2 compares injection behaviors, HIV/HCV risk perceptions, and HIV/HCV testing among PTO-First IDUs and other heroin IDUs. A higher proportion of PTO-First IDUs reported recent injection in their home or in a public restroom, injecting with friends, and recently getting new syringes from a spouse, family member, or sex partner. A higher proportion also were aware of San Diego's syringe exchange program and had used this program within the past three months; despite this, there was no significant difference in the proportion of PTO-First IDUs who reported receptive syringe sharing compared with other heroin IDUs. With regard to perceptions regarding $\mathrm{HIV} / \mathrm{HCV}$ risk, it was more common for PTO-First IDUs to perceive that they were at lower risk of HIV infection

Table I Demographic characteristics and drug use histories of PTO-First IDUs and other heroin IDUs

\begin{tabular}{|c|c|c|c|c|}
\hline & $\begin{array}{l}\text { Total \% } \\
(n=123)\end{array}$ & $\begin{array}{l}\text { PTO-first IDUs \% } \\
(n=49)\end{array}$ & $\begin{array}{l}\text { Other heroin IDUs \% } \\
(n=74)\end{array}$ & $P$ value \\
\hline Male & 74.8 & 79.6 & 71.6 & 0.319 \\
\hline Median age (IQR) & $29(22-32)$ & $27(24-33)$ & $31(24-34)$ & 0.296 \\
\hline \multicolumn{5}{|l|}{ Race/ethnicity } \\
\hline White non-Hispanic & 52.9 & 65.3 & 46.0 & \multirow[t]{2}{*}{0.035} \\
\hline Hispanic, Black, other & 47.1 & 34.7 & 54.1 & \\
\hline Higher than high school education & 34.2 & 44.9 & 27.0 & $0.04 I$ \\
\hline Income $\geq \$ 10,000$ past 12 months & 25.4 & 32.7 & 20.6 & 0.132 \\
\hline Lived with parents past 6 months & 30.3 & 41.7 & 23.0 & 0.028 \\
\hline \multicolumn{5}{|l|}{ Noninjection drug use (past 3 months) } \\
\hline Marijuana or hashish & 64.2 & 73.5 & 58.1 & 0.082 \\
\hline Methamphetamine & 47.2 & 42.9 & 50.0 & 0.437 \\
\hline Heroin & 53.7 & 69.4 & 43.2 & 0.004 \\
\hline Powder cocaine & 43.9 & 63.3 & 31.1 & $<0.001$ \\
\hline \multicolumn{5}{|l|}{ Injection drug use } \\
\hline Median age at first injection (IQR) & $19(17-23)$ & $21(18-26)$ & $18(17-22)$ & 0.013 \\
\hline Years since first injection (IQR) & $7(2-13)$ & $3(2-10)$ & $10(5-13)$ & $<0.001$ \\
\hline \multicolumn{5}{|l|}{ First drug injected } \\
\hline Heroin alone & 63.4 & 75.5 & 55.4 & \multirow[t]{3}{*}{0.062} \\
\hline Heroin combined with other drug & 8.9 & 4.1 & 12.2 & \\
\hline Stimulant/other & 27.6 & 20.4 & 32.4 & \\
\hline \multicolumn{5}{|l|}{ Drugs injected (past 3 months) } \\
\hline Heroin and cocaine together & 30.1 & 32.7 & 28.4 & 0.613 \\
\hline Heroin and meth together & 22.8 & 18.4 & 25.7 & 0.344 \\
\hline Methamphetamine by itself & 31.7 & 18.4 & 40.5 & 0.010 \\
\hline Heroin by itself & 79.7 & 85.7 & 75.7 & 0.176 \\
\hline Powder cocaine by itself & 21.1 & 22.5 & 20.3 & 0.772 \\
\hline
\end{tabular}

Abbreviation: IQR, interquartile range. 
Table 2 Injection behavior ${ }^{\mathrm{a}}$ and HIV/HCV risk perceptions and testing among PTO-First IDUs and other heroin IDUs.

\begin{tabular}{|c|c|c|c|c|}
\hline & $\begin{array}{l}\text { Total } \% \\
(n=123)\end{array}$ & $\begin{array}{l}\text { PTO-first IDUs \% } \\
(n=49)\end{array}$ & $\begin{array}{l}\text { Other heroin IDUs \% } \\
(n=74)\end{array}$ & $P$ value \\
\hline \multicolumn{5}{|l|}{ Injection locations } \\
\hline Your home & 65.6 & 77.6 & 57.5 & 0.023 \\
\hline Somebody else's home & 52.5 & 61.2 & 46.6 & 0.112 \\
\hline Shooting gallery & 7.4 & 2.0 & 11.0 & 0.065 \\
\hline Alleyway & 17.2 & 12.2 & 20.6 & 0.234 \\
\hline Street & 31.2 & 28.6 & 32.9 & 0.615 \\
\hline Park & 21.3 & 24.5 & 19.2 & 0.482 \\
\hline Public restroom & 45.9 & 57.1 & 38.4 & 0.041 \\
\hline \multicolumn{5}{|l|}{ Persons injected with } \\
\hline Friends & 66.9 & 81.6 & 56.9 & 0.005 \\
\hline Spouse & 9.1 & 8.2 & 9.7 & 0.770 \\
\hline Sex partner & 16.5 & 14.3 & 18.1 & 0.584 \\
\hline Acquaintance & 14.1 & 10.2 & 16.7 & 0.315 \\
\hline Drug dealer & 24.8 & 32.7 & 19.4 & 0.099 \\
\hline Strangers & 6.6 & 4.1 & 8.3 & 0.356 \\
\hline Alone & 49.6 & 44.9 & 52.8 & 0.395 \\
\hline \multicolumn{5}{|l|}{ Sources of new syringes } \\
\hline Pharmacy & 23.0 & 24.5 & 21.9 & $0.74 I$ \\
\hline Spouse/family/sex partner & 7.4 & 14.3 & 2.7 & 0.017 \\
\hline Friend & 47.5 & 51.0 & 45.2 & 0.528 \\
\hline Drug dealer & 22.1 & 30.6 & 16.4 & 0.064 \\
\hline SEP & 29.5 & 30.6 & 28.8 & 0.827 \\
\hline Receptive syringe sharing & 48.0 & 44.9 & 50.0 & 0.579 \\
\hline Aware of San Diego SEP & 82.9 & 91.8 & 77.0 & 0.033 \\
\hline Used SEP & 50.0 & 61.2 & 42.5 & 0.042 \\
\hline Fear police will interfere with drug use & 68.3 & 79.6 & 60.8 & 0.028 \\
\hline \multicolumn{5}{|l|}{ Likelihood of HIV compared to other IDUs } \\
\hline Less likely & 57.9 & 60.4 & 39.4 & 0.074 \\
\hline Same & 29.4 & 20.8 & 35.2 & \\
\hline More likely & 22.7 & 18.8 & 25.4 & \\
\hline Ever tested for HIV before study & 68.6 & 61.2 & 73.6 & 0.150 \\
\hline Tested HIV-positive in study & 0.8 & 0.0 & 1.4 & 0.587 \\
\hline \multicolumn{5}{|c|}{ Likelihood of HCV compared to other IDUs } \\
\hline Less likely & 36.4 & 51.0 & 26.4 & 0.021 \\
\hline Same & 30.6 & 22.5 & 36.1 & \\
\hline More likely & 33.1 & 26.5 & 37.5 & \\
\hline Ever tested for HCV before study & 49.5 & 54.4 & 46.0 & 0.391 \\
\hline Tested HCV-positive in study & 26.8 & 16.3 & 33.8 & 0.064 \\
\hline
\end{tabular}

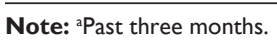

Abbreviations: SEP, syringe exchange program; HIV, human immunodeficiency virus; HCV, hepatitis C virus; IDUs, injection drug users; PTO, prescription-type opioid.

than other IDUs and a lower proportion had been tested for HIV, although these differences did not reach statistical significance. Similarly, it was more common for PTO-First IDUs to perceive themselves at lower risk of HCV infection, and the proportion testing positive for HCV was half that of other heroin IDUs.

Table 3 presents the results of the multiple logistic regression analysis. PTO-First IDUs had significantly higher odds of using noninjected powder cocaine in the past three months, injecting with friends in the past three months, getting new syringes from their spouse, family or sex partner in the past three months, and being aware of the San Diego syringe exchange program. They had significantly lower odds of methamphetamine injection over the past three months. Regarding HIV/HCV risk perceptions and testing, PTO-First IDUs had higher odds of perceiving themselves as less likely to be infected with HIV than other IDUs and lower odds of being tested for HIV prior to the study. There was no statistically significant difference in recruitment method across the two groups and there were no interactions between model variables or between the model variables and gender. 
Table 3 Factors independently associated with problematic prescription-type opioid use prior to heroin injection ${ }^{\mathrm{a}-\mathrm{c}}$

\begin{tabular}{|c|c|c|}
\hline & $\begin{array}{l}\text { Unadjusted } \\
\text { OR }(95 \% \mathrm{Cl})\end{array}$ & $\begin{array}{l}\text { Adjusted } \\
\text { OR }(95 \% \mathrm{Cl})\end{array}$ \\
\hline Noninjected powder cocaine ${ }^{d}$ & $3.82(1.78,8.18)$ & $3.75(1.43,9.86)^{\mathrm{e}}$ \\
\hline \multicolumn{3}{|l|}{ by itself ${ }^{d}$} \\
\hline Injected with friends ${ }^{d}$ & $3.36(1.42,7.95)$ & $6.01(1.90,19.07)^{\mathrm{e}}$ \\
\hline $\begin{array}{l}\text { Got syringes from spouse/ } \\
\text { family/sex partner }\end{array}$ & $5.92(1.17,29.8)$ & $23.0(2.33,226.0)^{\mathrm{e}}$ \\
\hline Aware of San Diego SEP & $3.36(1.05,10.67)$ & $7.28(1.17,45.05)$ \\
\hline \multicolumn{3}{|c|}{ Likelihood of HIV versus other IDUs } \\
\hline Same/not applicable & 1.00 & 1.00 \\
\hline Less likely & $2.59(1.05,6.36)$ & $4.32(1.26,14.77)$ \\
\hline More likely & $1.25(0.42,3.70)$ & $1.56(0.37,6.64)$ \\
\hline $\begin{array}{l}\text { Ever tested for HIV } \\
\text { before study }\end{array}$ & $0.57(0.26,1.23)$ & $0.25(0.08,0.80)$ \\
\hline $\begin{array}{l}\text { Notes: a Controlled for recruitme } \\
P<0.05 \text {; ' } m o d e l \text { Chi-square stat } \\
\text { Bonferroni adjustment for multiple } \\
\text { Abbreviations: OR, odds ratio }\end{array}$ & ite and all other facto & $\begin{array}{l}\text { in the table; ball values } \\
\text { onths; }{ }^{e}<0.05 \text { after } \\
\text { EP, syringe exchange }\end{array}$ \\
\hline
\end{tabular}

\section{Conclusion}

The transition from prescription-type opioid use to heroin is a phenomenon that has been described in the US mainstream media, but is as yet largely unstudied in the scientific literature. In this study of young heroin IDUs in San Diego, California, we found that $40 \%$ reported problematic use of prescription-type opioids prior to initiating heroin use. Similar proportions of prior problematic prescription-type opioid use have been reported in studies of young heroin IDUs in Portland, Oregon (47\%) and Seattle, Washington (44\%). ${ }^{12,13}$ The findings from these three cities, in combination with previously published reports, ${ }^{6-9,14}$ indicate an urgent need for prospective studies that 1) provide a detailed characterization of transitions from prescription-type opioid use to heroin use, particularly heroin injection, 2) identify the factors that facilitate or impede these transitions, and 3) propose appropriate interventions to prevent these transitions.

We found that PTO-First IDUs were sociodemographically different from other heroin IDUs, because a higher proportion of PTO-First IDUs were white non-Hispanic, had education beyond high school, and recently resided with their parents. In addition, our study suggests that the drug use networks of PTO-First IDUs may differ markedly from those of other heroin IDUs. In the multivariate analysis, PTO-First IDUs had higher odds of injecting with friends and getting new syringes from a spouse, family member, or sex partner. Moreover, in the univariate analysis, a higher proportion of prescription-type opioid-first IDUs reported injecting at home and injecting in a public restroom. A number of harm reduction interventions have targeted injection drug user networks. ${ }^{8}$ Future studies should determine whether PTO-First injection networks differ from those of other heroin IDUs and, if so, what the implications are for network-based HIV/HCV prevention interventions. The role of social networks in facilitating or impeding transitions from prescription-type opioids to heroin use and injection also requires further exploration.

We also found significant differences between the two groups with regard to HIV/HCV risk perceptions. Although a similar proportion of PTO-First IDUs and other heroin IDUs reported receptive syringe sharing, and PTO-First IDUs had higher odds of perceiving themselves at lower risk of HIV infection than other IDUs. They also had significantly lower odds of ever having had an HIV test. Perceptions regarding $\mathrm{HCV}$ risk trended in the same direction but did not reach statistical significance in the multivariate model. As noted, PTO-First IDUs had higher odds of injecting with friends; they may believe that they know their injection partners better, and/or that their injection partners have a low likelihood of infection, thus putting themselves at lower risk. Admittedly, the proportion of participants testing positive for HIV in both groups of heroin IDUs was very low and the proportion of PTO-First IDUs testing positive for $\mathrm{HCV}$ was half that of other heroin IDUs; if PTO-First IDUs tend to inject among themselves, their social networks might present a lower risk of HIV/HCV transmission than other heroin IDUs. However, whether these IDUs actually know the status of their injecting partners is not clear, and a substantial percentage of IDUs in our study had never been tested for HIV or HCV. Further, changes in the injection networks of PTO-First IDUs that involve contact with HIV-positive or HCV-positive injectors could quickly increase their risk of infection. It should be noted that PTO-First IDUs had been injecting for a median of three years compared with a median of seven years among other heroin IDUs. It may be that this shorter duration of injection underlies both the lower proportion of $\mathrm{HCV}$ infections and lower perception of HIV/HCV infection risk among PTO-First IDUs rather than any substantial differences in risky injection behaviors or injection networks. Nonetheless, understanding the structure and dynamics of PTO-First IDUs injection networks could provide critical information upon which to develop peer-based interventions to address $\mathrm{HIV} / \mathrm{HCV}$ risk perceptions and reduce associated risk behaviors. 
Our study did find that PTO-First IDUs engage in injection behaviors that may put them at risk of HIV and HCV. Almost half reported sharing syringes, more than half reported injecting in public spaces (eg, public restrooms), and a significantly higher proportion of PTO-First IDUs said they feared police interference with their drug use. Both public injecting and fear of police involvement have been associated with risky injection practices. ${ }^{15-21}$ In addition, public injection has been associated with a higher risk of drug overdose, ${ }^{22,23}$ and fear of police has been associated with reduced likelihood of calling 911 in response to a drug overdose, ${ }^{24-27}$ which may put PTO-First IDUs at disproportionate risk of overdose and related mortality. Accordingly, understanding why PTO-First IDUs perceive themselves to be at lower risk of infection with blood-borne pathogens and the implications for morbidity and mortality related to HIV, HCV, overdose, and other adverse health effects are significant public health priorities for this subpopulation of IDUs.

Certain limitations must be considered while interpreting these findings. This was an unfunded exploratory study that was nested in a larger study examining recruitment methods for HCV studies among IDUs. The parent study provided an efficient vehicle to investigate problematic prescriptiontype opioid use as a precursor to heroin use among heroin injectors, but it was not designed to generate a generalizable estimate of the prevalence of prior problematic prescriptiontype opioid use in this population. Accordingly, our findings might not be generalizable to all PTO-First IDUs. However, the use of three different recruitment methods should have produced a combined sample that has greater representation than samples from a single method, and the finding of almost identical proportions of PTO-First IDUs in studies in Portland and Seattle imply that more epidemiologically rigorous studies of prevalence may generate similar results. We also acknowledge that the way problematic prescriptiontype opioid use was assessed (ie, “...were you hooked on prescription-type opiates?") does not adhere to DSM-IV (Diagnostic and Statistical Manual of Mental Disorders, Fourth Edition) definitions of abuse and dependence, and leaves uncharacterized the exact nature of prescription-type opioid use prior to heroin initiation. The wording of this question was selected because it is identical to wording used in the Portland and Seattle studies, which allowed for comparison across the three regions, ${ }^{12,13}$ and interviewers in those two cities reported that the question was accurately understood by interviewees and had good face validity.
Finally, since the parent study was not originally designed to assess prescription-type opioid use, certain useful questions were not included (eg, reasons for initiating heroin use and whether prescription-type opioids were prescribed versus obtained illicitly). We also did not collect information on current prescription-type opioid use, which would provide insights on whether PTO-First IDUs are distinguishable from other heroin IDUs based on their current opioid use. Despite these limitations, our findings provide valuable preliminary data for furthering the research agenda regarding the role of prescription-type opioid use in pathways toward heroin injection, as well as the reasons underlying low perceptions of HIV/HCV risk among PTO-First IDUs and the related health implications.

In summary, we found that a high proportion of young heroin IDUs reported problematic use of prescription-type opioids prior to initiating heroin use and that these PTOFirst IDUs differ from other heroin IDUs with regard to early and recent drug use, injection networks, and HIV risk perceptions and testing experience, but not with regard to syringe sharing. Our study contributes to the very small body of literature on the role of prescription-type opioids as a precursor to heroin use and injection, and identifies important areas for future study.

\section{Acknowledgment}

Funding for the parent study was provided by the Centers for Disease Control and Prevention. The findings and conclusions in this report are those of the authors and do not necessarily represent the views of the Centers for Disease Control and Prevention.

\section{Disclosure}

The authors report no conflicts of interest in this work.

\section{References}

1. Substance Abuse and Mental Health Services Administration. Results from the 2009 National Survey on Drug Use and Health: Volume I. Summary of National Findings (Office of Applied Studies, NSDUH Series H-38A, HHS Publication No. SMA 10-4856). Rockville, MD. Available at: http://oas.samhsa.gov/NSDUH/2k9NSDUH/2k9Results.htm\#Ch2. Accessed July 23, 2011.

2. Johnston LD, O’Malley PM, Bachman JG, Schulenberg JE. Monitoring the Future National Survey results on drug use, 1975-2010. Volume I: Secondary school students. Ann Arbor, MI: Institute for Social Research, The University of Michigan. Available at: http://www monitoringthefuture.org. Accessed July 23, 2011.

3. Substance Abuse and Mental Health Services Administration. Substance Abuse and Mental Health Data Archive: Quick Tables for the Treatment Episode Data Set -Admissions (TEDS-A) - Concatenated, 1992 to present. Available at: http://www.icpsr.umich.edu/quicktables/quickconfig do?25221-20001. Accessed July 23, 2011. 
4. Substance Abuse and Mental Health Services Administration. Detailed Tables: National Estimates, Drug-Related Emergency Department Visits for 2004-2009. Available at: http://dawninfo.samhsa.gov/data/. Accessed July 23, 2011.

5. Centers for Disease Control and Prevention. QuickStats: number of poisoning deaths involving opioid analgesics and other drugs or substances - United States, 1999-2007. Available at: http://www.cdc. gov/mmwr/preview/mmwrhtml/mm5932a6.htm?s_cid=mm5932a6_e. Accessed September 12, 2011.

6. Siegal HA, Carlson RG, Wang J, Falck RS, Stephens RC, Nelson ED. Injection drug users in the Midwest: an epidemiologic comparison of drug use patterns in four Ohio cities. J Psychoactive Drugs. 1994;26(3): 265-275.

7. Daniulaityte RD, Carlson RG, Kenne D. Initiation to pharmaceutical opioids and patterns of misuse: Preliminary qualitative findings obtained by the Ohio Substance Abuse Monitoring Network. J Drug Issues. 2006; 4(36):787-808.

8. Inciardi JA, Surratt HL, Cicero TJ, Beard RA. Prescription opioid abuse and diversion in an urban community: the results of an ultrarapid assessment. Pain Med. 2009;10(3):537-548.

9. Lankenau SE, Teti M, Silva K, Bloom JJ, Harocopos A, Treese M. Initiation into prescription opioid misuse amongst young injection drug users. Int J Drug Policy. June 19, 2011. [Epub ahead of print.]

10. Garfein RS, Rondinelli A, Barnes RFW, et al. Modest HCV prevalence despite risky injection practices among injection drug users in San Diego, California. Oral presentation at the National HIV Prevention Conference, Atlanta, GA, August 14-17, 2011.

11. Heckathorn DD. Respondent-driven sampling: A new approach to the study of hidden populations. Soc Prob. 1997:44(2):174-199.

12. Pollini RA, Banta-Green C, Jenkins L, Teshale E, Garfein R. High prevalence of prescription opioid use preceding heroin use in three west coast cities. Oral late-breaker presentation at the 73rd Annual Meeting of the College on Problems of Drug Dependence, June 18-23, 2011, Hollywood, FL.

13. Banta-Green C, Jackson TR, Albert D, et al. Drug abuse trends in the Seattle/King County Area: 2009. In: Epidemiological Trends in Drug Abuse: Proceedings of the Community Epidemiology Work Group, Volume II. June 9-11, 2010. Boston, MA: Department of Health and Human Services; 2011.

14. Grau LE, Dasgupta N, Harvey AP, et al. Illicit use of opioids: is OxyContin a "gateway drug"? Am J Addict. 2007;16(3):166-173.

15. Latkin CA, Sherman S, Knowlton A. HIV prevention among drug users: outcome of a network-oriented peer outreach intervention. Health Psychol. 2003;22(4):332-339.

16. Latkin CA, Mandell W, Vlahov D, Oziemkowska M, Celentano DD. The long-term outcome of a personal network-oriented HIV prevention intervention for injection drug users: the SAFE Study. Am J Community Psychol. 1996;24(3):341-364.
17. Tobin KE, Kuramoto SJ, Davey-Rothwell MA, Latkin CA. The STEP into Action study: a peer-based, personal risk network-focused HIV prevention intervention with injection drug users in Baltimore, Maryland. Addiction. 2011;106(2):366-375.

18. Aitken C, Moore D, Higgs P, Kelsall J, Kerger M. The impact of a police crackdown on a street drug scene: evidence from the street. Int J Drug Policy. 2002;13(3):193-202.

19. Bluthenthal RN, Lorvick J, Kral AH, Erringer EA, Kahn JG. Collateral damage in the war on drugs: HIV risk behaviors among injection drug users. Int J Drug Policy. 1999;10(1):25-38.

20. Cooper H, Moore L, Gruskin S, Krieger N. The impact of a police drug crackdown on drug injectors' ability to practice harm reduction: a qualitative study. Soc Sci Med. 2005;61(3):673-684.

21. Dovey K, Fitzgerald J, Choi Y. Safety becomes danger: dilemmas of drug-use in public space. Health Place. 2001;7(4):319-331.

22. Marshall BD, Kerr T, Qi J, Montaner JSG, Wood E. Public injecting and HIV risk behaviour among street-involved youth, Drug Alcohol Depend. 2010;110(3):254-258.

23. Rhodes T, Mikhailova L, Sarang A, et al. Situational factors influencing drug injecting, risk reduction and syringe exchange in Togliatti City, Russian Federation: a qualitative study of micro risk environment. Soc Sci Med. 2003;57(1):39-54.

24. Small W, Rhodes T, Wood E, Kerr T. Public injection settings in Vancouver: physical environment, social context and risk. Int J Drug Policy. 2007;18(1):27-36.

25. Dietze P, Jolley D, Fry C, Bammer G. Transient changes in behavior lead to heroin overdose: results from a case-crossover study of non-fatal overdose. Addiction. 2005;100(5):636-642.

26. Darke S, Kaye S, Ross J. Geographical injecting locations among injection drug users in Sydney, Australia. Addiction. 2001;96(2):241-246.

27. Davidson PJ, Ochoa KC, Hahn JA, Evans JL, Moss AR. Witnessing heroin-related overdoses: the experiences of young injectors in San Francisco. Addiction. 2002;97(12):1511-1516.

28. Tracy M, Piper TM, Ompad D, et al. Circumstances of witnessed drug overdose in New York City: implications for intervention. Drug Alcohol Depend. 2005;79(2):181-190.

29. Darke S, Ross J, Hall W. Overdose among heroin users in Sydney, Australia: II. Responses to overdose. Addiction. 1996;91(3):413-417.

30. Pollini RA, McCall L, Mehta SH, Celentano DD, Vlahov D, Strathdee SA. Response to overdose among injection drug users. Am J Prev Med. 2006;31(3):261-264.
Substance Abuse and Rehabilitation

\section{Publish your work in this journal}

Substance Abuse and Rehabilitation is an international, peer-reviewed, open access journal publishing original research, case reports, editorials, reviews and commentaries on all areas of addiction and substance abuse and options for treatment and rehabilitation. The manuscript management system is completely online and includes a very quick and fair

\section{Dovepress}

peer-review system. Visit http://www.dovepress.com/testimonials.php to read real quotes from published authors. 